\title{
Surface-modified fibrous membranes for fuel cell application
}

\author{
Noor Fatina Emelin Nor Fadzil1, Ebrahim Abouzari-Lotf ${ }^{1,2, *}$, Mohan V. Jacob ${ }^{3}$, \\ Nurfatehah Wahyuny Che Jusoh ${ }^{4}$, and Arshad Ahmad ${ }^{1,2}$ \\ ${ }^{1}$ Advanced Material Research Group, Center of Hydrogen Energy, Institute of Future Energy, \\ Universiti Teknologi Malaysia, International Campus, 54100 Kuala Lumpur, Malaysia. \\ ${ }^{2}$ Department of Chemical Engineering, School of Chemical and Energy Engineering, Faculty of \\ Engineering, Universiti Teknologi Malaysia, 81310 Johor Bahru, Johor, Malaysia. \\ ${ }^{3}$ Electronics Materials Lab, College of Science, Technology and Engineering, James Cook University, \\ Townsville, QLD 4811, Australia. \\ ${ }^{4}$ Malaysia-Japan International Institute of Technology, Universiti Teknologi Malaysia, Jalan Sultan \\ Yahya Petra, 54100 Kuala Lumpur, Malaysia.
}

\begin{abstract}
Low permeability layers of poly(1-vinylimidazole) were polymerised and deposited onto both sides of electrospun polyethersulfone (PES) nanofibrous sheet radiofrequency plasma. The layers not only act as an efficient fuel barrier layer but also impart high and stable proton conductivity, as well as better chemical and dimensional stabilities. Typically, the composite membrane exhibited methanol permeability as low as $33.20 \times 10^{-8} \mathrm{~cm}^{2} \mathrm{~s}^{-1}$ and high through-plane proton conductivity of 52.4 $\mathrm{mS} \mathrm{cm}{ }^{-1}$ at $95 \% \mathrm{RH}$, indicating membrane selectivity of $0.675 \times 10^{8} \mathrm{mS} . \mathrm{s}$ $\mathrm{cm}^{-3}$, which is approximately 33 times greater than the selectivity of N115 under similar conditions.
\end{abstract}

Keywords: Poly(1-vinyl imidazole), electrospun nanofibres, plasma, composite membranes, proton transfer

\section{Introduction}

In order to improve energy efficiency and durability of polymer electrolyte fuel cells (PEFCs), the development of proton exchange membranes (PEMs) must be conducted. Progress in PEMs will also help in refining the performance of other electrochemical energy systems including polymer-ion and redox flow batteries (RFBs), not limited to the enhancement in fuel cell only [1-3]. Typically, high proton conductivity together with low fuel permeability and adequate mechanical immovability are required for PEMs as they have different functions of separating reactants and conducting protons while electrically insulating [4-6]. Characteristically, proton conductivity of perfluorinated sulfonic acid (PFSA) membranes is remarkable when used in direct methanol fuel cells (DMFCs).

\footnotetext{
*Corresponding author: ebrahim@utm.my
} 
However, high methanol permeability results in fuel loss, as well as lower fuel cell performance and energy efficiency [7]. In fact, the expected higher volumetric energy density for DMFC and direct ethanol fuel cell (DEFC) compared to a hydrogen fuel cell cannot be achieved due to such undesired methanol crossover through commercially available membranes. Such limitation along with the sluggish kinetics of methanol or ethanol electrooxidation keeps such devices far away from their theoretical potentials.

The solution to the limitations of high fuel permeability without seriously affecting the desired proton conductivity or stability is by modifying commercially available membranes or developing new membrane materials. The most widely used method is by using inorganic fillers to retard feed permeability. In addition to pristine inorganic fillers, various functionalised fillers contain simple functional groups; also, amine, amide, and imidazole are also used to subdue the fuel permeability of membranes [4, 8-9]. The addition of such fillers restructures hydrophilic channels, which reduces fuel permeability by increasing the tortuosity of diffusion. Although there are improvements in terms of the dispersion and hydrophilic nature of the filler by functionalisation with various acidic groups [10], the key challenge in preparing hybrid membranes is to reach unvarying dispersion of filler materials and its compatibility with the polymer matrix [11].

An alternative solution is to use an impermeable or low-permeable layer in the surface of the membrane to reduce the high crossover of membrane. The impermeable layers can be added on the membrane by graft polymerisation using electron-beam, $\gamma$-ray, and ultraviolet light irradiation or by plasma [11]. This method provides rapid development of active sites on polymer membranes and it is easy to be used. In addition, it provides excellent consistency, depth control, simple preparation, and low penetrability [12].

In this study, polyethersulfone (PES) fibrous sheet was modified by polymerisation and deposition of poly(1-vinylimidazole) on the surface using radiofrequency plasma. The morphology and microstructure of specimens were characterised by atomic force microscopy (AFM), scanning electron microscopy (SEM), and Fourier transform infrared spectroscopy (FTIR).

\section{Experimental methods}

\subsection{Characterisation}

SEM images of the composite membranes were attained using Philips XL30 field emission scanning electron microscope (FESEM) after coating with $5 \mathrm{~nm} \mathrm{Au}$. The surface roughness of the membrane was determined using Bruker Innova AFM. Agilent Cary 660 spectrometer was used to perform FTIR analysis.

The through-plane and in-plane proton conductivity of membrane was assessed under several relative humidity conditions using four-point probe of Bekk Tech conductivity cell (BT-112). A specific voltage was set between two inner probes and the resulting current was measured. The resistance (R) was obtained by calculating the slope of the current versus the voltage measurement graph, and proton conductivity $(\sigma)$ was calculated using the following equation:

$$
\sigma(S / \mathrm{cm})=\frac{L(\mathrm{~cm})}{\left(\left(R(\Omega) \times A(\mathrm{~cm})^{2}\right)\right.}
$$


Where $\mathrm{A}$ is the area of the membrane, $\mathrm{L}$ is the distance in the direction of the ion flow between voltage measurement probes, and the denominator term of equation, $\mathrm{R}(\Omega) \times \mathrm{A}(\mathrm{cm})^{2}$, describes area resistance [6].

Permeability of methanol (P) was calculated from the slope of the plot between $\mathrm{C}_{\mathrm{R}}$ and diffusion time (t) using Equation 2:

$$
C_{R}=\frac{A}{V_{R}} \frac{P}{L} C_{o}\left(t-t_{o}\right)
$$

Where $V_{R}$ is the diffusion reservoir volume, $t_{0}$ and $C_{0}$ are the time lag and the initial concentration of methanol in the compartment on the left side of the membrane, respectively, and $\mathrm{L}$ and $\mathrm{A}$ are the thickness and area of the membrane, respectively [6].

\subsection{Production of composite membranes}

Electroris (FNM Ltd.) system was employed for electrospinning production of PES (RADEL 300, Amaco Performance Products Inc., OH, USA). Solutions containing 25 wt. \% of PES were prepared in dimethylacetamide (DMAC) under constant stirring rate in a glass reactor at room temperature. $15.2 \mathrm{kV}$ of voltage was applied and the distance was set at $17 \mathrm{~cm}$ between the tip of the metal needle and the collector. The flow rate was set at $0.8 \mathrm{ml} \mathrm{h}^{-1}$ for the polymer solutions and controlled by a syringe pump. The rotational speed of the collector drum was set at $300 \mathrm{rpm}$. After $7 \mathrm{~h}$ of electrospinning, the materials with the desired thickness were carefully removed and placed in a vacuum oven for $12 \mathrm{~h}$.

Poly(1-vinylimidazole) thin film with approximate thickness of $20 \mathrm{~nm}$ was deposited on both sides of PES fibre using a custom-made tubular plasma polymerisation apparatus at input power of $50 \mathrm{~W}$.

\section{Results and discussion}

\subsection{Microscopy analysis of PES nanofibre and plasma-modified membranes}

Morphology of the electrospun substrates was determined from high (a) and low (b)

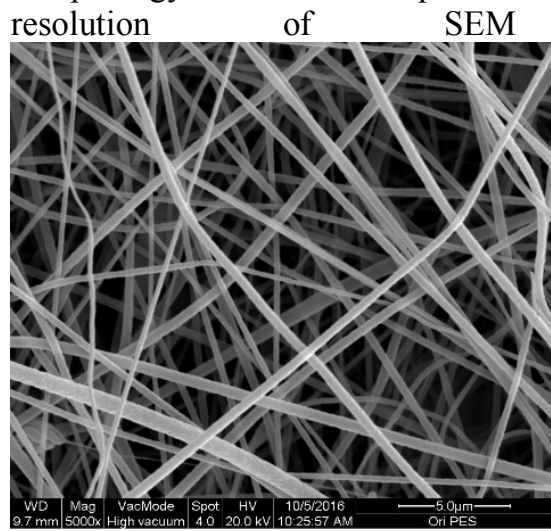

(a)

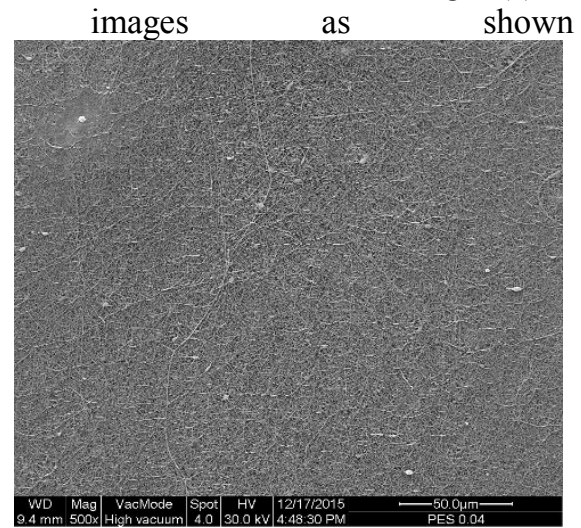

(b) 
Fig. 1.

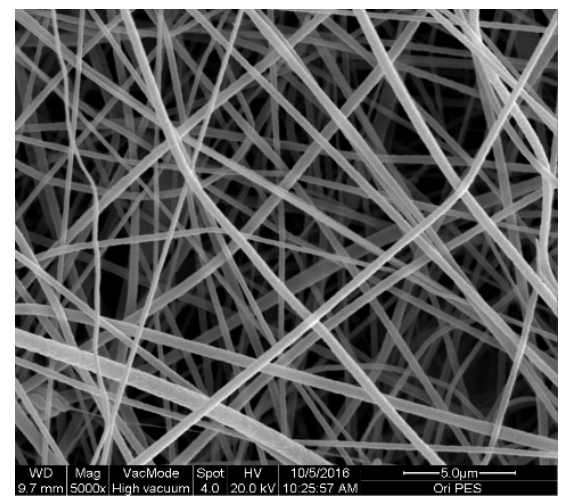

(a)

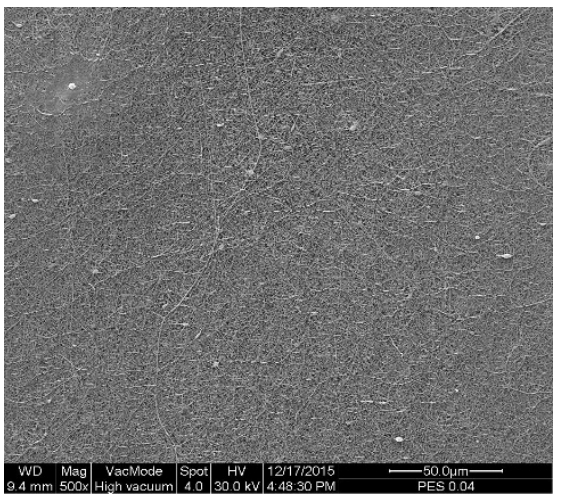

(b)

Fig. 1. a) High and b) low resolution of SEM images showing morphology of electrospun PES.

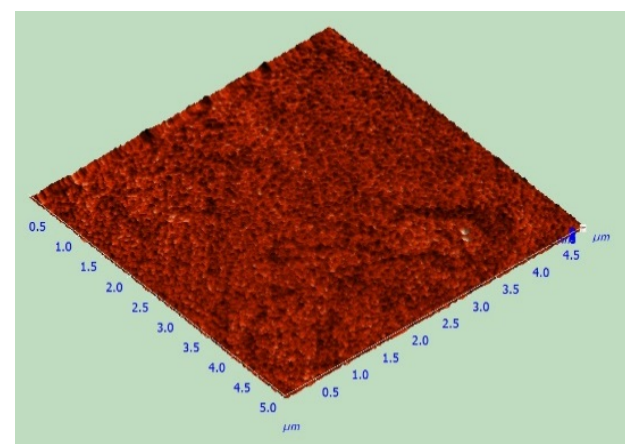

Fig. 2. AFM image for modified PES membrane.

Fig. 2 shows the AFM image of plasma-modified PES. Surface roughness of the membrane was determined from AFM analysis. By using software from Bruker Innova PES membrane with $50 \mathrm{~W}$ and thickness of $20 \mathrm{~nm}$, the surface roughness was obtained and recorded at an average of $0.465 \mathrm{~nm}$.

\subsection{FTIR analysis of PES, poly(1-vinylimidazole), and modified PES membranes}

The IR spectrum of PES substrate in Fig. 3 shows C-H aromatic stretching at $3082 \mathrm{~cm}^{-1}$ and $\mathrm{CH}_{3}$ asymmetric aliphatic stretching at $2907 \mathrm{~cm}^{-1}$ [13]. Also, in-plane skeletal benzene rings were observed at $1070 \mathrm{~cm}^{-1}$ and ring vibration of $p$-substituted aryl ether was detected at 1010 $\mathrm{cm}^{-1}[14]$.

Meanwhile, the IR spectrum for poly(1-vinylimidazole) illustrates seven main peaks at 3326 and $2934 \mathrm{~cm}^{-1}$ corresponding to absorbed water and C-H ring stretching, and 1652 and $1456 \mathrm{~cm}^{-1}$ for $\mathrm{C}-\mathrm{H}$ ring stretching and ring bending. The peak at $1226 \mathrm{~cm}^{-1}$ signals $\mathrm{C}-\mathrm{H}$ chain 
bending and $\mathrm{C}-\mathrm{N}$ ring stretching, the peak at $750 \mathrm{~cm}^{-1}$ corresponds to $\mathrm{C}-\mathrm{N}$ ring stretching and chain bending, and the peak at $661 \mathrm{~cm}^{-1}$ represents the ring torsion of poly(1-vinyl imidazole).

A ratio of peaks at several positions of the IR spectra across modified plasma PES membrane was determined and the original IRs of PES and poly(1-vinylimidazole) was compared. The modified PES peak ratio at $3298 \mathrm{~cm}^{-1}$ and $2928 \mathrm{~cm}^{-1}$ was 0.92 . By comparing these peaks with PES and poly(1-vinylimidazole) around the same wavelength, it was found that the modified PES membrane contained traces of poly(1-vinylimidazole) as the ratio was closer to poly(1-vinylimidazole). Another observed peak ratio of 1.03 was obtained at 687 $\mathrm{cm}^{-1}$ and $668 \mathrm{~cm}^{-1}$, and the results confirmed the traces of poly(1-vinylimidazole) of 1.73 compared to 0.03 for PES.

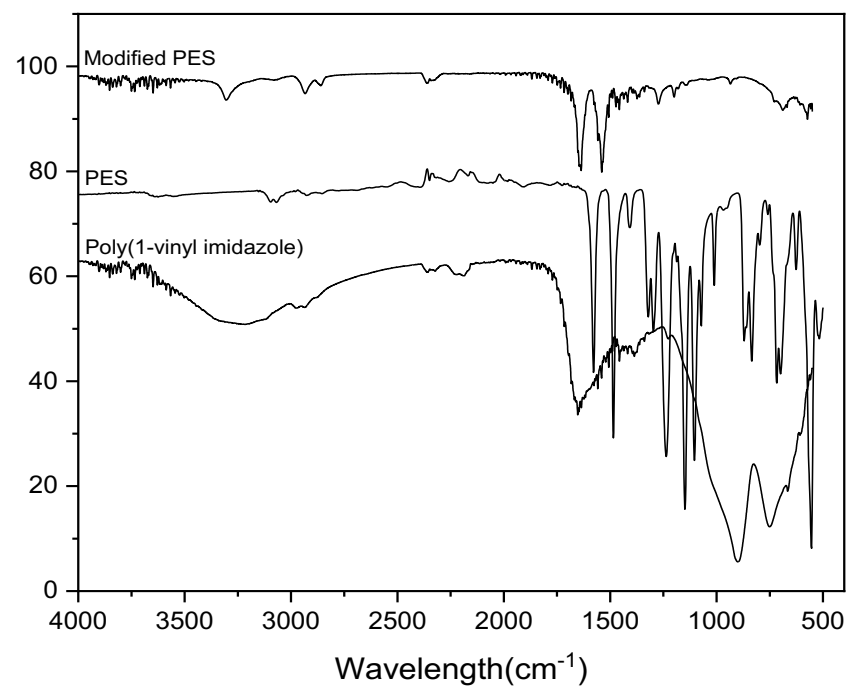

Fig. 3. FTIR spectra of combined original PES, poly(1-vinylimidazole), and modified PES membranes.

\subsection{Proton conductivity and methanol permeability of plasma-modified PES membranes}

Proton conductivity for plasma-modified PES membranes was evaluated under $95 \%$ relative humidity $(\mathrm{RH})$ at 30 and $80{ }^{\circ} \mathrm{C}$, and at $20 \% \mathrm{RH}$ and $80{ }^{\circ} \mathrm{C}$. The conductivities of the developed membranes under $95 \% \mathrm{RH}$ at 30 and $80{ }^{\circ} \mathrm{C}$ were 22.4 and $52.4 \mathrm{mS} \mathrm{cm}^{-1}$, respectively. Meanwhile, at $20 \% \mathrm{RH}$ and $80{ }^{\circ} \mathrm{C}$, the recorded conductivity was $0.9 \mathrm{mS} \mathrm{cm}{ }^{-1}$. The proton conductivities of commercial membrane $\mathrm{N} 115$ at $95 \% \mathrm{RH}$ and 30 and $80{ }^{\circ} \mathrm{C}$ were 52.9 and $154.1 \mathrm{mS} \mathrm{cm}^{-1}$, respectively. At $20 \% \mathrm{RH}$ and $80{ }^{\circ} \mathrm{C}$, the recorded conductivity was $3.6 \mathrm{mS} \mathrm{cm}^{-1}$. By comparing the results of the conductivity, permeability, and selectivity to commercial membrane N115, the conductivity of the modified PES membrane was slightly lower than N115; however, the methanol permeability for the modified membrane was greatly reduced from $104 \times 10^{-8} \mathrm{~cm}^{2} \mathrm{~s}^{-1}$ for N115 to $33.20 \times 10^{-8} \mathrm{~cm}^{2} \mathrm{~s}^{-1}$. The selectivity of the modified PES was also improved from $0.509 \times 10^{8} \mathrm{mS} . \mathrm{s} \mathrm{cm}^{-3}$ for N115 to $0.675 \times 10^{8}$ $\mathrm{mS} . \mathrm{s} \mathrm{cm}^{-3}$.

\section{Conclusion}


To formulate proton-conductive and low-methanol permeable membrane for DMFC, poly(1vinylimidazole) was plasma-polymerised and deposited onto both sides of electrospun PES nanofibrous sheet. The additional layers not only act as an effective fuel barrier layer but also promote stable and high proton conductivity. The chemical and dimensional stabilities will also be improved by these additional layers. The composite membrane displayed high through-plane proton conductivity of $52.4 \mathrm{mS} \mathrm{cm}^{-1}$ and methanol permeability as low as $33.20 \times 10^{-8} \mathrm{~cm}^{2} \mathrm{~s}^{-1}$ at $95 \% \mathrm{RH}$. The membrane selectivity of $0.675 \times 10^{8} \mathrm{mS} \cdot \mathrm{s} \mathrm{cm}^{-3}$ indicates that the selectivity of composite membrane is 33 times greater than the selectivity of commercial membrane N115. This upgraded performance together with the outstanding selectivity and stability, untraceable leaching of poly(1-vinylimidazole), and subsequent durability of conductivity represent the prospective of these composite membranes as an influential candidate for applications at higher fuel concentrations.

The research was primarily supported by a joint partnership research programme of Innovative Research Universities of Australia and Malaysia Research University Network (IRU-MRUN) (Vot. No. 00M84) and MTJA No. 4C116.

\section{References}

[1] C. Guo, L. Zhou, and J. Lv, 'Effects of expandable graphite and modified ammonium polyphosphate on the flame-retardant and mechanical properties of wood flourpolypropylene composites', Polym. Polym. Compos., vol. 21, no. 7, pp. 449-456, 2013.

[2] M. M. Nasef, 'Radiation-grafted membranes for polymer electrolyte fuel cells: Current trends and future directions', Chem. Rev., vol. 114, no. 24, pp. 12278-12329, 2014.

[3] K.-D. Kreuer, 'Ion Conducting Membranes for Fuel Cells and other Electrochemical Devices', Chem. Mater., vol. 26, no. 1, pp. 361-380, 2013.

[4] E. Abouzari-Lotf, M. Etesami, and M. M. Nasef, Carbon-Based Nanocomposite Proton Exchange Membranes for Fuel Cells. Elsevier Inc., 2018.

[5] M. Zakeri, E. Abouzari-Lotf, M. M. Nasef, A. Ahmad, M. Miyake, T. M. Ting, and P. Sithambaranathan, 'Fabrication and characterization of supported dual acidic ionic liquids for polymer electrolyte membrane fuel cell applications', Arab. J. Chem., 2018.

[6] E. Abouzari-Lotf, M. M. Nasef, H. Ghassemi, M. Zakeri, A. Ahmad, and Y. Abdollahi, 'Improved Methanol Barrier Property of Nafion Hybrid Membrane by Incorporating Nanofibrous Interlayer Self-Immobilized with High Level of Phosphotungstic Acid', ACS Appl. Mater. Interfaces, vol. 7, no. 31, pp. 17008-17015, 2015.

[7] X. Li and A. Faghri, 'Review and advances of direct methanol fuel cells (DMFCs) part I: Design, fabrication, and testing with high concentration methanol solutions', J. Power Sources, vol. 226, pp. 223-240, 2013.

[8] A. A. Argun, J. N. Ashcraft, and P. T. Hammond, 'Highly conductive, methanol resistant polyelectrolyte multilayers', Adv. Mater., vol. 20, no. 8, pp. 1539-1543, 2008.

[9] J. Rozière and D. J. Jones, 'Non-Fluorinated Polymer Materials for Proton Exchange Membrane Fuel Cells', Annu. Rev. Mater. Res., vol. 33, no. 1, pp. 503-555, 2003.

[10] V. Parthiban, S. Akula, and A. K. Sahu, 'Surfactant templated nanoporous carbon-Nafion hybrid membranes for direct methanol fuel cells with reduced methanol crossover', J. Memb. Sci., vol. 541, pp. 127-136, Nov. 2017.

[11] E. Y. Choi, H. Strathmann, J. M. Park, and S. H. Moon, 'Characterization of non-uniformly charged ion-exchange membranes prepared by plasma-induced graft polymerization', $J$. Memb. Sci., vol. 268, no. 2, pp. 165-174, 2006.

[12] S.-C. Liao, K.-S. Chen, W.-Y. Chen, C.-Y. Chou, and K.-C. Wai, 'Surface Graft Polymerization of Acrylamide onto Plasma Activated Nylon Microfiber Artificial Leather for Improving Dyeing Properties', Int. J. Chem. Eng. Appl., vol. 4, no. 2, pp. 78-81, 2013.

[13] S. Belfer, R. Fainchtain, Y. Purinson, and O. Kedem, 'Surface characterization by FTIRATR spectroscopy of polyethersulfone membranes-unmodified, modified and protein 
fouled', J. Memb. Sci., vol. 172, no. 1-2, pp. 113-124, 2000.

[14] M. R. Pereira and J. Yarwood, 'ATR-FTIR spectroscopic studies of the structure and permeability of sulfonated poly(ether sulfone) membranes. Part 1.-Interfacial water-polymer interactions', J. Chem. Soc. Faraday Trans., vol. 92, no. 15, pp. 2731-2735, 1996. 